\title{
Relativistic and non-relativistic analysis of whistler-mode waves in a hot anisotropic plasma
}

\author{
By S. S. SAZHIN, A. E. SUMNER \\ Department of Physics, The University of Sheffield, Sheffield S3 7RH, U.K. \\ AND N. M. TEMME \\ Centrum voor Wiskunde en Informatica, Kruislaan 413, 1098 SJ Amsterdam, \\ The Netherlands
}

(Received 7 November 1991 and in revised form 16 December 1991)

The dispersion equation for parallel whistler-mode propagation in a hot anisotropic plasma is analysed numerically in both weakly relativistic and nonrelativistic approximations under the assumption that wave growth or damping does not influence the wave refractive index. The results of this analysis are compared with the results of an asymptotic analysis of the same equation, and the range of applicability of the latter results is specified. It is pointed out that relativistic effects lead to a decrease in the range of frequencies for which instability occurs. For a moderately anisotropic plasma $\left(T_{\perp} / T_{\|}=2\right)$ relativistic effects lead to an increase in the maximum value of the increment of instability.

\section{Introduction}

Storey (1953) was the first to show that the dispersion of electromagnetic signals generated during lightning discharges and propagating through the earth's magnetosphere could be explained in terms of the theory of electromagnetic wave propagation in a cold plasma. These signals are known as whistlers, and the mode in which they propagate through the magnetosphere is known as the whistler -mode (Helliwell 1965). It has since been recognized that these waves are responsible for the transfer of wave energy from artificial radio transmitters and many types of natural radio emissions (Sazhin 1982; Bullough 1983; Carpenter 1988). Moreover, it is now generally believed that these waves play an important role in the loss of electrons from the radiation belts in the earth's magnetosphere (Kennel \& Petschek 1966) and presumably in the magnetospheres of other planets (see e.g. Coroniti et al. 1987; Gurnett et al. 1990). They can exist in the atmospheres of stars and in laboratory plasmas (see e.g. Wharton \& Trivelpiece 1966). They have been observed in metals (Kaner \& Skobov 1971) and semiconductors (Baynham \& Boardman 1971), where they are known as helicon waves. This accounts for the attention that researchers in different fields of physics pay to this particular type of wave.

Since the pioneering paper by Storey (1953), there have been different developments in the theory of whistler-mode waves, In particular, attempts have been made to take into account both non-zero electron temperature and anisotropy of the electron distribution function and their effects on whistler- 
mode propagation, growth or damping (see e.g. Kennel \& Petschek 1966; Sazhin $1988 a, 1991)$. In most cases the theory of whistler-mode propagation in a hot anisotropic plasma has been based on the non-relativistic approximation, i.e. on a self-consistent solution of the linearized Maxwell equations and the non-relativistic Vlasov equation. This approximation was believed to be applicable with confidence to magnetospheric conditions, which satisfy

$$
w \ll c,
$$

where $w$ is the thermal velocity of electrons and $c$ is the velocity of light. At the same time, most of those who developed this theory seem to have turned a blind eye to (or simply had not noticed) the fact that the non-relativistic approach to the analysis of waves in a hot plasma is not self-consistent, since it neglects relativistic effects in the Vlasov equation but takes them into account in the Maxwell equations, which are relativistic by their nature.

An alternative approach to the theory of whistler-mode waves in a hot plasma has been based on the so-called weakly relativistic approximation, which uses the condition (1) with terms of order $w^{2} / c^{2}$ taken into account, allowing substantial simplification of the general relativistic wave dispersion equation (see e.g. Shkarofsky 1966 ; Jacquinot \& Leloup 1971; Sazhin 1987 a). However, even this relatively simple weakly relativistic whistler-mode dispersion equation is much more complicated than the non-relativistic dispersion equation, and its applications have been very limited (Jacquinot \& Leloup 1971; Wingle 1983; Robinson 1987a).

In a series of papers (Sazhin $1987 a, b, 1989$; Sazhin \& Temme 1990, $1991 a, b$ ) we have attempted to develop an asymptotic theory of whistler-mode propagation, instability or damping in a weakly relativistic plasma. Restricting ourselves to the limiting, but practically important, case of whistler-mode propagation strictly parallel to the magnetic field, we obtained some approximate solutions to the weakly relativistic dispersion equation. These solutions are rather simple, and we hope that they will be widely used in the plasma physics community. The main restriction of these solutions is that we cannot always be sure about their range of applicability, just as we cannot always be sure about the range of applicability of solutions of the nonrelativistic dispersion equation.

In order to specify the range of applicability of different approximate solutions of the weakly relativistic dispersion equation, as well as those of the non-relativistic equation, we need to compare them with the results of a rigorous numerical analysis of the weakly relativistic whistler-mode dispersion equation. It is this comparison that we make here. As in the papers by Sazhin $(1987 b, 1989)$ and Sazhin \& Temme $(1990,1991 a, b)$, we restrict ourselves to parallel whistler-mode waves. This paper can be considered as complementary to that of Sazhin $(1988 b)$, where a comparison between asymptotic nonrelativistic and weakly relativistic solutions was made, but different types of waves propagating almost parallel and almost perpendicular to the external magnetic field were considered.

The basic weakly relativistic and non-relativistic dispersion equations and their approximate solutions are presented in $\$ 2$. The results of the comparison of different solutions are shown and discussed in $\$ 3$. The main results of the paper are summarized in $\S 4$. 


\section{Basic equations}

The dispersion equation for parallel whistler-mode waves in a weakly relativistic (with condition (1) valid) anisotropic plasma can be written as (Sazhin \& Temme 1990)

where

$$
N^{2}=1-\frac{2 X}{r}\left[\mathscr{F}_{\frac{1}{2}, 2}-\frac{d \mathscr{F}_{\frac{3}{2}, 2}}{d z}\left(A_{e}-1\right) N^{2}\right]
$$

$$
\begin{gathered}
\mathscr{F}_{q, p} \equiv \mathscr{F}_{q, p}(z, a, b)=-i \int_{0}^{\infty} \exp \left(i z t-\frac{a t^{2}}{1-i t}\right)(1-i t)^{-q}(1-i b t)^{-p} d t \\
z=\frac{2(1-Y)}{r}, \quad a=\frac{N^{2}}{r}, \quad r=\frac{p_{0 \|}^{2}}{m_{e}^{2} c^{2}}, \\
b=A_{e}, \quad X=\frac{\Pi_{0}^{2}}{\omega^{2}}, \quad Y=\frac{\Omega_{0}}{\omega}, \quad A_{e}=\frac{p_{0 \perp}^{2}}{p_{0 \|}^{2}} .
\end{gathered}
$$

$\Pi_{0}, \Omega_{0}$ and $\omega$ are the electron plasma frequency at rest, the electron gyrofrequency at rest and the wave frequency (which is complex in general) respectively, $N \equiv c k / \omega$ is the wave refractive index, $m_{e}$ is the electron mass at rest, $c$ is the velocity of light and $k$ is the wavenumber. In deriving (2) we have assumed an electron distribution function of the form

$$
f\left(p_{\perp}, p_{\|}\right)=\left(j ! \pi^{\frac{3}{2}} p_{0 \perp}^{2 j+2} p_{0 \|}\right)^{-1} p_{\perp}^{2 j} \exp \left(-\frac{p_{\perp}^{2}}{p_{0 \perp}^{2}}-\frac{p_{\|}^{2}}{p_{0 \|}^{2}}\right),
$$

where $p_{0 \perp(\|)}$ is the electron thermal momentum in the direction perpendicular (parallel) to the magnetic field, $p_{\perp}$ and $p_{\|}$are the electron momenta in the corresponding directions, and $j=0,1,2, \ldots$.

We have restricted our analysis to the case $j=0$ (a bi-Maxwellian plasma). The generalization to $j \neq 0$ would be straightforward (Tsai et al. 1981; Sazhin 1989). We have also restricted ourselves to considering wave frequencies well above the proton gyrofrequency, so that the protons and other heavy ions can be considered as a neutralizing background.

We call the function $\mathscr{F}_{q, p}$ the generalized Shkarofsky function. It is related to the conventional Shkarofsky function (Shkarofsky 1966; Robinson 1986, $1987 b$ )

$$
\mathscr{F}_{q}(z, a)=-i \int_{0}^{\infty} \exp \left(i z t-\frac{a t^{2}}{1-i t}\right)(1-i t)^{-q} d t
$$

by the identities

$$
\begin{gathered}
\mathscr{F}_{q, 0}(z, a, b)=\mathscr{F}_{q, p}(z, a, 0)=\mathscr{F}_{q}(z, a), \\
\mathscr{F}_{q, p}(z, a, 1)=\mathscr{F}_{q+p}(z, a) .
\end{gathered}
$$

In the non-relativistic limit $c \rightarrow \infty$ the expression for the generalized Shkarofsky function simplifies to

$$
\mathscr{F}_{q, p}=-\frac{1}{2 a^{\frac{1}{2}}} Z\left(\frac{z}{2 a^{\frac{1}{2}}}\right)
$$


where $Z \equiv Z(\xi)$ is the non-relativistic plasma dispersion function, given by

$$
Z(\xi)=i \pi^{\frac{1}{2}} \exp \left(-\xi^{2}\right)-2 \int_{0}^{\xi} \exp \left(-\xi^{2}+t^{2}\right) d t
$$

In view of (2) and (6), the non-relativistic parallel whistler-mode dispersion equation can be written as

$$
N^{2}=1+\left(A_{e}-1\right) X+\left(X / N r^{\frac{1}{2}}\right)\left[A_{e}+\left(1-A_{e}\right) Y\right] Z(\xi),
$$

where $\xi=z / 2 a^{\frac{1}{2}}$.

Solutions to (2) and (8) are greatly simplified if we restrict our analysis to the case of weakly growing or weakly damped waves, i.e.

$$
\gamma \equiv \operatorname{Im}(\omega) \ll \min \left(\omega_{0}, \Omega_{0}-\omega_{0}\right),
$$

where $\omega_{0} \equiv \operatorname{Re}(\omega)$. The condition (9) is in fact not restrictive for applications of our theory. If it is violated, we cannot neglect nonlinear effects, which was done in deriving (2) and (8).

In view of (9), we can present the complex equation (2) as a system of two equations :

$$
\begin{gathered}
N^{2}=1-\frac{2 X}{r}\left[\operatorname{Re}\left(\mathscr{F}_{\frac{1}{2}, 2}\right)-\frac{d \operatorname{Re}\left(\mathscr{F}_{\frac{3}{2}, 2}\right)}{d z}\left(A_{e}-1\right) N^{2}\right], \\
\tilde{\gamma} \equiv \frac{\gamma}{\omega}=\frac{X\left\{\operatorname{Im}\left(\mathscr{F}_{\frac{1}{2}, 2}\right)\left[1+\left(A_{e}-1\right) N^{2}\right]-\left(A_{e}-1\right) N^{2} \operatorname{Im}\left(\mathscr{F}_{\frac{3}{2}, 2}\right)\right\}}{r\left[1+\left(2 X / r^{2}\right) \sum_{i=0}^{3} \tilde{a}_{i} \operatorname{Re}\left(\mathscr{F}_{i-\frac{1}{2}, 2}\right)\right]},
\end{gathered}
$$

where

$$
\begin{gathered}
\tilde{a}_{0}=Y+N^{2}\left[1+Y\left(A_{e}-1\right)\right]+N^{4}\left(A_{e}-1\right), \\
\tilde{a}_{1}=\left(A_{e}-1\right) N^{2} r-Y-2 N^{2}\left[1+Y\left(A_{e}-1\right)\right]-3 N^{4}\left(A_{e}-1\right), \\
\tilde{a}_{2}=-\left(A_{e}-1\right) N^{2} r+N^{2}\left[1+Y\left(A_{e}-1\right)\right]+3 N^{4}\left(A_{e}-1\right), \\
\tilde{a}_{3}=-\left(A_{e}-1\right) N^{4} .
\end{gathered}
$$

(To simplify the notation, we shall henceforth assume that $\omega \equiv \operatorname{Re}(\omega) \equiv \omega_{0}$.) Equation (10) describes wave propagation, while (11) describes wave growth $(\tilde{\gamma}>0)$ or damping $(\tilde{\gamma}<0)$ in a weakly relativistic plasma.

In a similar way we can reduce the complex non-relativistic dispersion equation (8) to the following system of equations:

$$
\begin{gathered}
N^{2}=1+\left(A_{e}-1\right) X+\left(X / N r^{\frac{1}{2}}\right)\left[A_{e}+\left(1-A_{e}\right) Y\right] \operatorname{Re}\left[Z\left(\xi_{0}\right)\right], \\
\tilde{\gamma} \equiv \frac{\gamma}{\omega}=\frac{-\pi^{\frac{1}{2}}\left[A_{e}-\left(A_{e}-1\right) Y\right] \exp \left(-\xi_{0}^{2}\right)}{2(1-Y) / X \xi_{0}+\kappa \xi_{0}+\operatorname{Re}\left[Z\left(\xi_{0}\right)\right]\left(A_{e}+\kappa \xi_{0}^{2}\right)},
\end{gathered}
$$

where

$$
\xi_{0} \equiv \operatorname{Re}(\xi), \quad \kappa=\frac{2\left[A_{e}+Y\left(1-A_{e}\right)\right]}{Y-1} .
$$

Equation (12) describes wave propagation, while (13) describes wave growth or damping in a hot anisotropic (but non-relativistic) plasma.

In the cold-plasma limit (as $\left|\xi_{0}\right| \rightarrow \infty$ ) (12) reduces to

$$
N=N_{00} \equiv\left(1+\frac{X}{Y-1}\right)^{\frac{1}{2}}
$$


while in the same limit (13) gives $\tilde{\gamma}=0$. This means that in a cold plasma whistler-mode waves propagate without damping or growth. In the limiting case $Y \gg 1$ and $X \gg 1$, (14) reduces to that derived and analysed by Storey (1953).

In the limit as $r \rightarrow 0$, but keeping terms of the order of $r$, we can write an approximate solution of (10) as (Jacquinot \& Leloup 1971; Sazhin 1987a,b)

$$
N=N_{00}\left[1+\frac{\beta_{e} Y^{3}}{2(Y-1)^{3}}+\frac{\beta_{e}\left(1+4 A_{e}\right) Y^{2}}{4(Y-1)^{2} N_{00}^{2}}-\frac{\beta_{e} A_{e} Y^{2}}{2(Y-1)^{2}}\right]
$$

where $\beta_{e}=\frac{1}{2} \nu r, \nu=\Pi_{0}^{2} / \Omega_{0}^{2}$ and $N_{00}$ is defined by (14). In the limit as $N_{00}^{2} \rightarrow \infty$ this expression reduces to that which could be derived from the corresponding nonrelativistic dispersion equation (12).

A similar simplification of (11) appears to be not so straightforward. So far it has been done only for the analysis of marginally stable waves for which $\tilde{\gamma}=0$ (Sazhin \& Temme $1991 a, b$ ). The results of the latter paper will be compared with numerical results in $\S 3$.

The non-relativistic expression for $\tilde{\gamma}$ can be considerably simplified if we neglect the effects of non-zero electron temperature on wave propagation (cf. Kennel \& Petschek 1966); it is then reduced to

where

$$
\tilde{\gamma}=\frac{\pi^{\frac{1}{2}} \xi_{00}(Y-1)\left[A_{e}-\left(A_{e}-1\right) Y\right] \exp \left(-\xi_{00}^{2}\right)}{2(1-Y)^{2} / X+Y},
$$

$$
\xi_{00}=\frac{1-Y}{N_{00} r^{\frac{1}{2}}} .
$$

The values of $N$ that follow from $(10),(12),(14)$ and (15) and the values of $\tilde{\gamma}$ that follow from (11), (13) and (16) will be compared in $\S 3$ for different values of the plasma parameters $\left(\nu, r\right.$ and $\left.A_{e}\right)$.

\section{Results}

In this section we compare numerical values of $N$ and $\tilde{\gamma}$ obtained from the approximations discussed in $\$ 2$ for different values of the parameters typical for conditions in the earths magnetosphere. In order to simplify this comparison, we introduce the parameters $N_{r}, N_{n}, N_{r a}, N_{00}, \tilde{\gamma}_{r}, \tilde{\gamma}_{n}$ and $\tilde{\gamma}_{n s}$, the meaning of which is specified in table 1 .

The numerical analyses of (10) and (12) were based on the method of bisection. The algorithm for computation of the generalized Shkarofsky functions is discussed elsewhere (Temme, Sumner \& Sazhin 1992). It is essential to note that our numerical code works only when $|z / 2 a|<1 / A_{e}$, i.e. it fails for a very rarefied and anisotropic plasma. However, this restriction seems to be not very important for magnetospheric applications.

First we consider a relatively hot $(r=0.01$, corresponding to $T \approx 2.5 \mathrm{keV})$ and dense $(\nu=5)$ plasma with anisotropy $A_{e}=2$. Plots of $N$ and $\tilde{\gamma}$ versus $\omega / \Omega_{0}$ $\left(\equiv Y^{-1}\right)$ for the different approximations for this plasma are shown in figures $1(a, b)$. As can be seen from figure $1(a)$, the values of $N_{r a}$ are closest to those of $N_{r}$ at $\omega / \Omega_{0} \lesssim 0 \cdot 6$. In any case, the approximation $N_{r a}$ is better than the nonrelativistic approximation $N_{n}$ at $\omega / \Omega_{0} \lesssim 0 \cdot 6$. The latter approximation is applicable for the qualitative analysis of $N$ only. The curves of $N$ versus $\omega / \Omega_{0}$ are henceforth presented only for those values of $\omega / \Omega_{0}$ for which $|\tilde{\gamma}| \lesssim 0 \cdot 2$. We 


\begin{tabular}{clc}
\hline Parameter & \multicolumn{1}{c}{ Meaning } & See equation \\
$N_{r}$ & Weakly relativistic refractive index & $(10)$ \\
$N_{n}$ & Non-relativistic refractive index & $(12)$ \\
$N_{r a}$ & Approximate expression for the weakly relativistic refractive & $(15)$ \\
& index & $(14)$ \\
$N_{00}$ & Refractive index in a cold plasma & $(11)$ \\
$\tilde{\gamma}_{r}$ & Weakly relativistic value of $\tilde{\gamma}$ & $(13)$ \\
$\tilde{\gamma}_{n}$ & Non-relativistic value of $\tilde{\gamma}$ & $(16)$ \\
$\tilde{\gamma}_{n s}$ & Simplified non-relativistic value of $\tilde{\gamma}$ & \\
& \multicolumn{2}{c}{ TABLE 1} \\
\hline
\end{tabular}

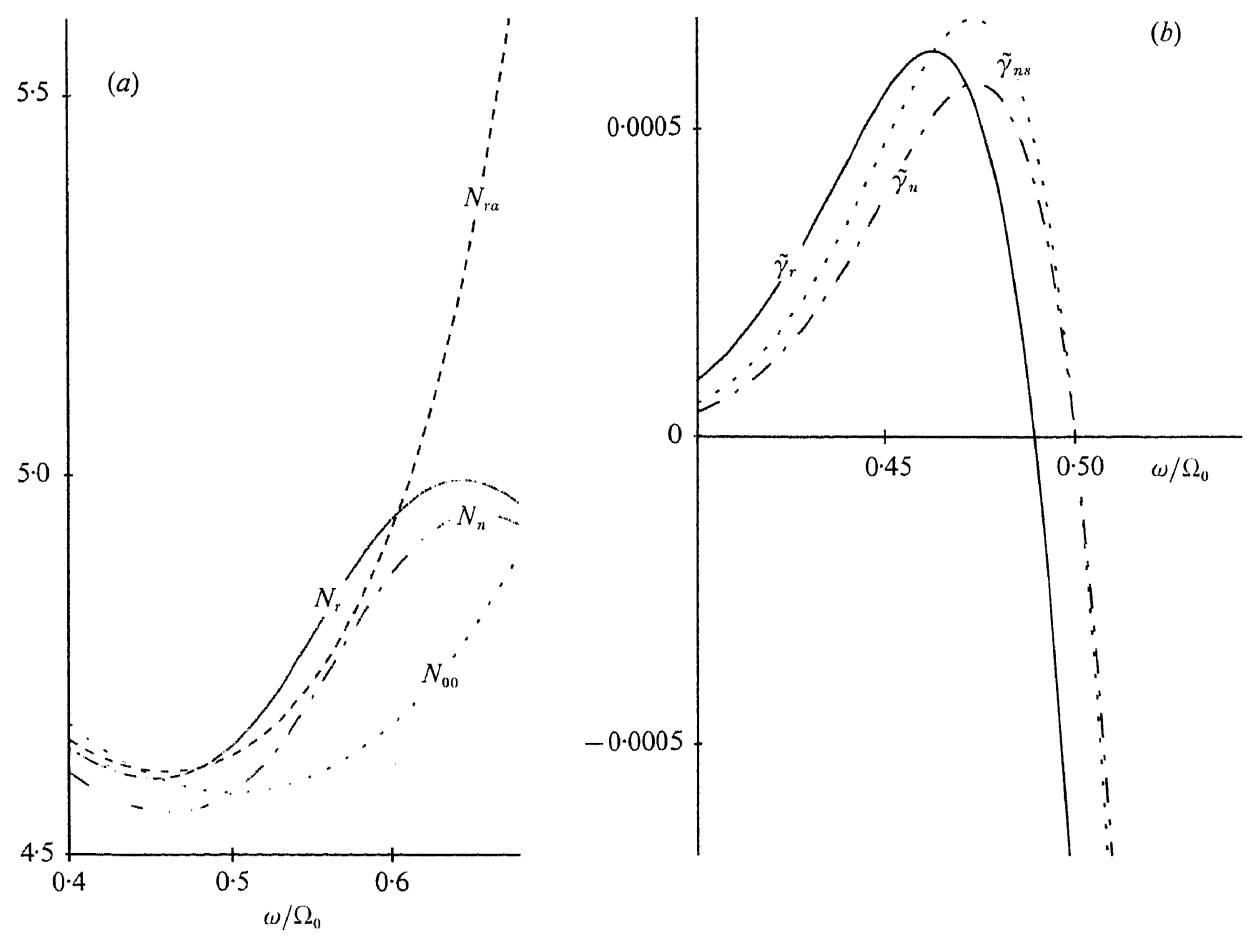

Figure 1. (a) Plots of $N_{r}\left(-\right.$, see (10)), $N_{n}\left(-.-.-\right.$, see (12)), $N_{r a}\left(---\right.$, see (15)) and $N_{00}$ $(\cdots$, see $(14))$ versus $\omega / \Omega_{0}\left(\equiv Y^{-1}\right)$ for a plasma with parameters $r=0 \cdot 01, \nu=5$ and $A_{e}=2$. Plots are shown only for those values of $\omega / \Omega_{0}$ for which $\tilde{\gamma}_{r, n, n s} \leqslant 0 \cdot 2$ (the condition (9) is satisfied). (b) Plots of $\tilde{\gamma}_{r}(-$, see $(11)), \tilde{\gamma}_{n}(-\ldots-\ldots$, see $(13))$ and $\tilde{\gamma}_{n s}(\cdots$, , see $(16))$ versus $\omega / \Omega_{0}$ for the same plasma parameters as in $(a)$.

have tried to choose values of the parameters $r, \nu$ and $A_{e}$ that are relevant to the conditions in the earth's magnetosphere. However, these parameters vary there over such a wide range that we were bound to restrict ourselves to some illustrative examples only.

As follows from figure $1(b)$, non-relativistic theory predicts whistler-mode instability in a slightly wider frequency range compared with weakly relativistic theory, although the peak value of $\left|\tilde{\gamma}_{n}\right|$ is slightly smaller than that of $\left|\tilde{\gamma}_{r}\right|$. The approximate expression $\tilde{\gamma}_{n s}$ gives slightly larger values of the increment of instability compared with $\tilde{\gamma}_{n}$. The frequency at which whistler-mode waves are 

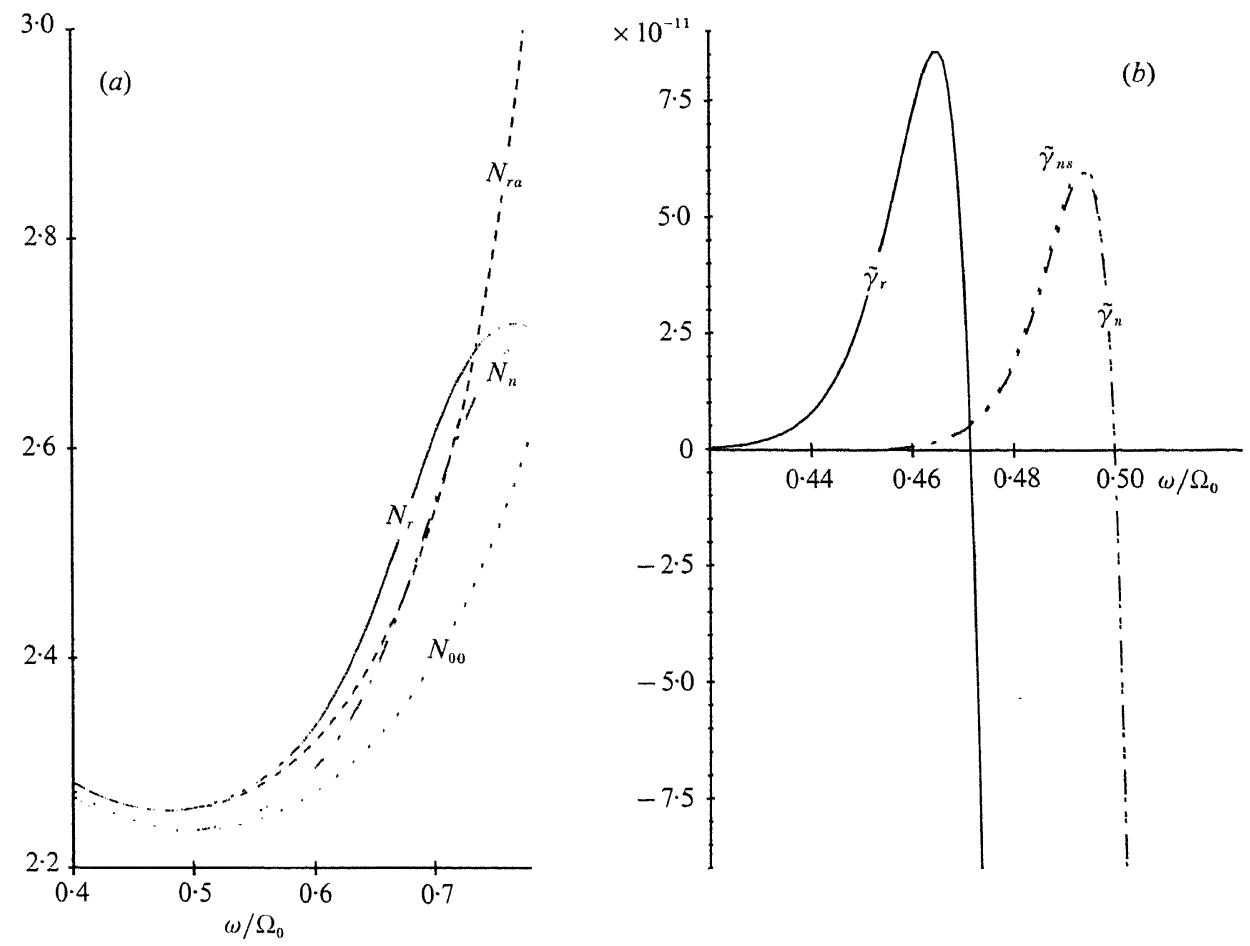

Figure 2. As figure 1, but for $\nu=1$.

marginally stable is approximately $0.490 \Omega_{0}$ in the weakly relativistic approximation, which is consistent with the asymptotic results of Sazhin \& Temme $(1991 b)$.

In figure $2(a, b)$ we show curves similar to those in figure $1(a, b)$, but for $\nu=1$. As follows from figure $2(a), N_{r a}$ can approximate $N_{r}$ at $\omega / \Omega_{0} \lesssim 0 \cdot 7$, and almost coincides with $N_{r}$ when $\omega / \Omega_{0} \lesssim 0.5$. The shapes of the curves for $N_{r}$ and $N_{n}$ are similar to those shown in figure $1(a)$, although the absolute values of both $N_{r}$ and $N_{n}$ are lower.

The curves for $\tilde{\gamma}_{n s}$ and $\tilde{\gamma}_{n}$ in figure $2(b)$ are closer to each other compared with those in figure $1(b)$, but the deviation between relativistic and non-relativistic curves is more pronounced in figure $2(b)$ than in $1(b)$. As in the case shown in figure $1(b)$, the relativistic theory predicts the development of an instability in a narrower frequency range than follows from the non-relativistic theory, but the peak value of $\tilde{\gamma}$ is larger for the relativistic curve. The frequency of marginal stability of whistler-mode waves is approximately $0 \cdot 471 \Omega_{0}$, which is again consistent with the results of the asymptotic analysis by Sazhin \& Temme $(1991 b)$.

In figure $3(a, b)$ we show curves similar to those in figures $1(a, b)$ and $2(a, b)$, but for $\nu=0 \cdot 5$. The main differences between the curves in figure $3(a)$ and those in figure $2(a)$ are (i) the curve $N_{r a}$ is close to $N_{r}$ in a wider frequency range (up to $\omega / \Omega_{0} \lesssim 0.78$; at $\omega / \Omega_{0} \lesssim 0.6$ the curves $N_{r a}$ and $N_{r}$ almost coincide); and (ii) the absolute values of $N$ shown in figure $3(a)$ are lower than those shown in figure $2(a)$.

The curves $\tilde{\gamma}_{n s}$ and $\tilde{\gamma}_{n}$ in figure $3(b)$ coincide within the accuracy of plotting, 


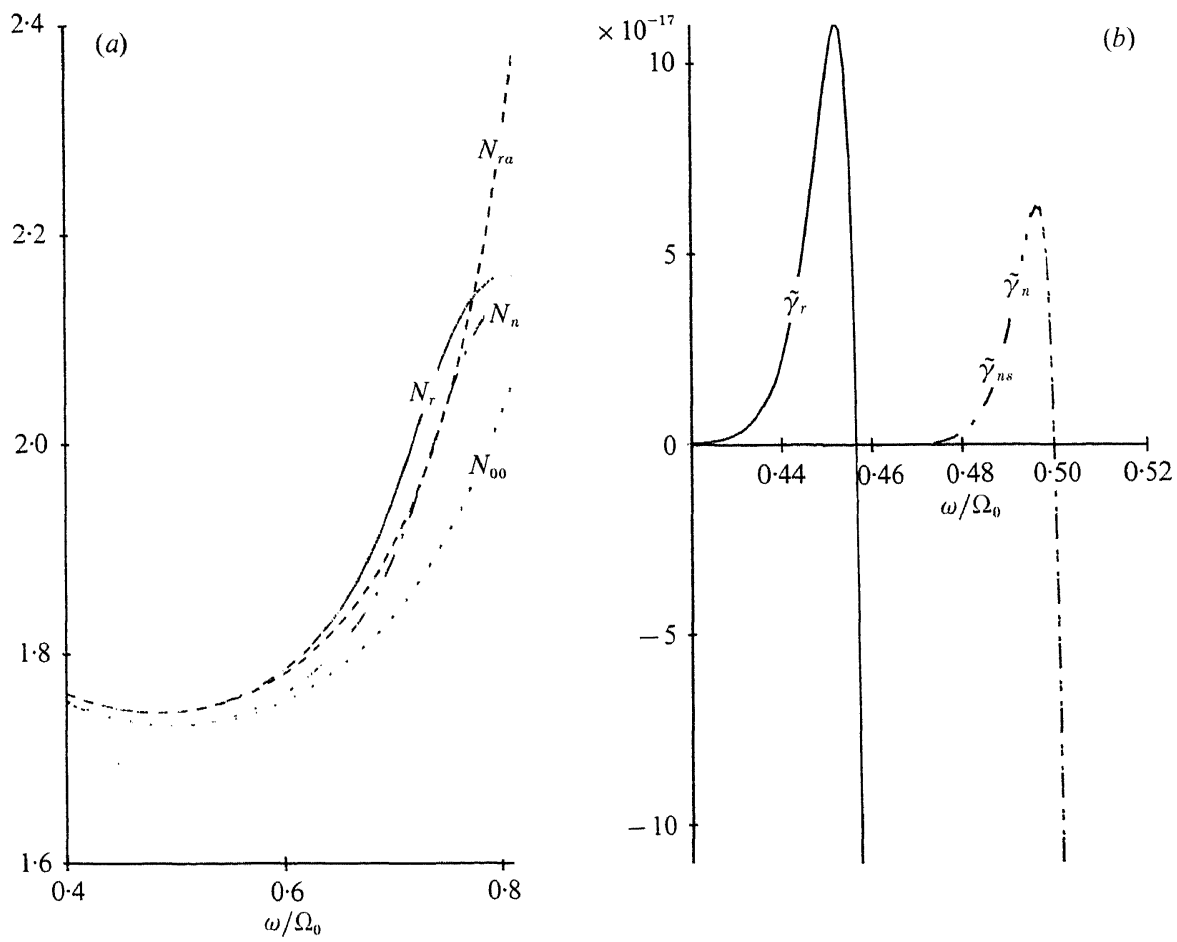

Figure 3. As figure 1, but for $\nu=0.5$.
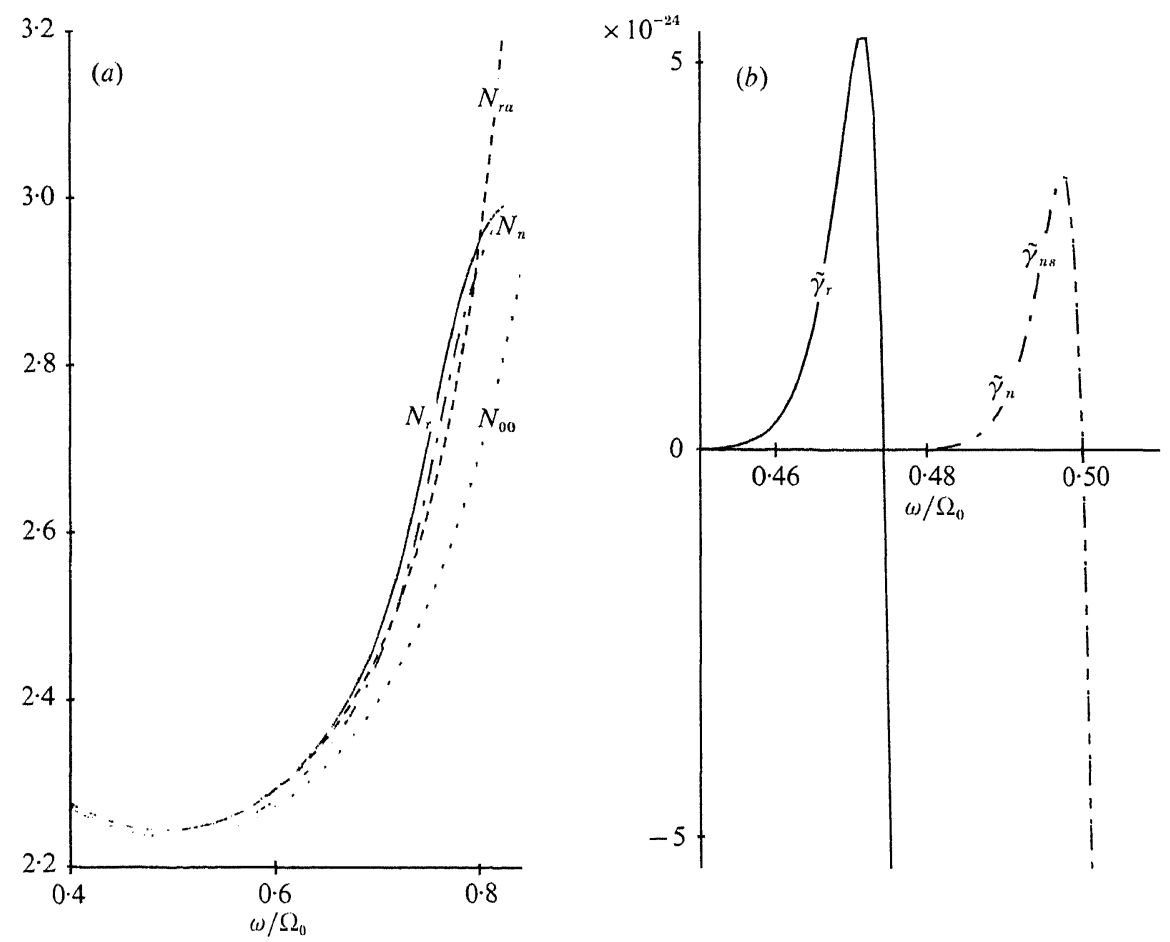

Figure 4. As figure 2, but for $r=0.004$. 

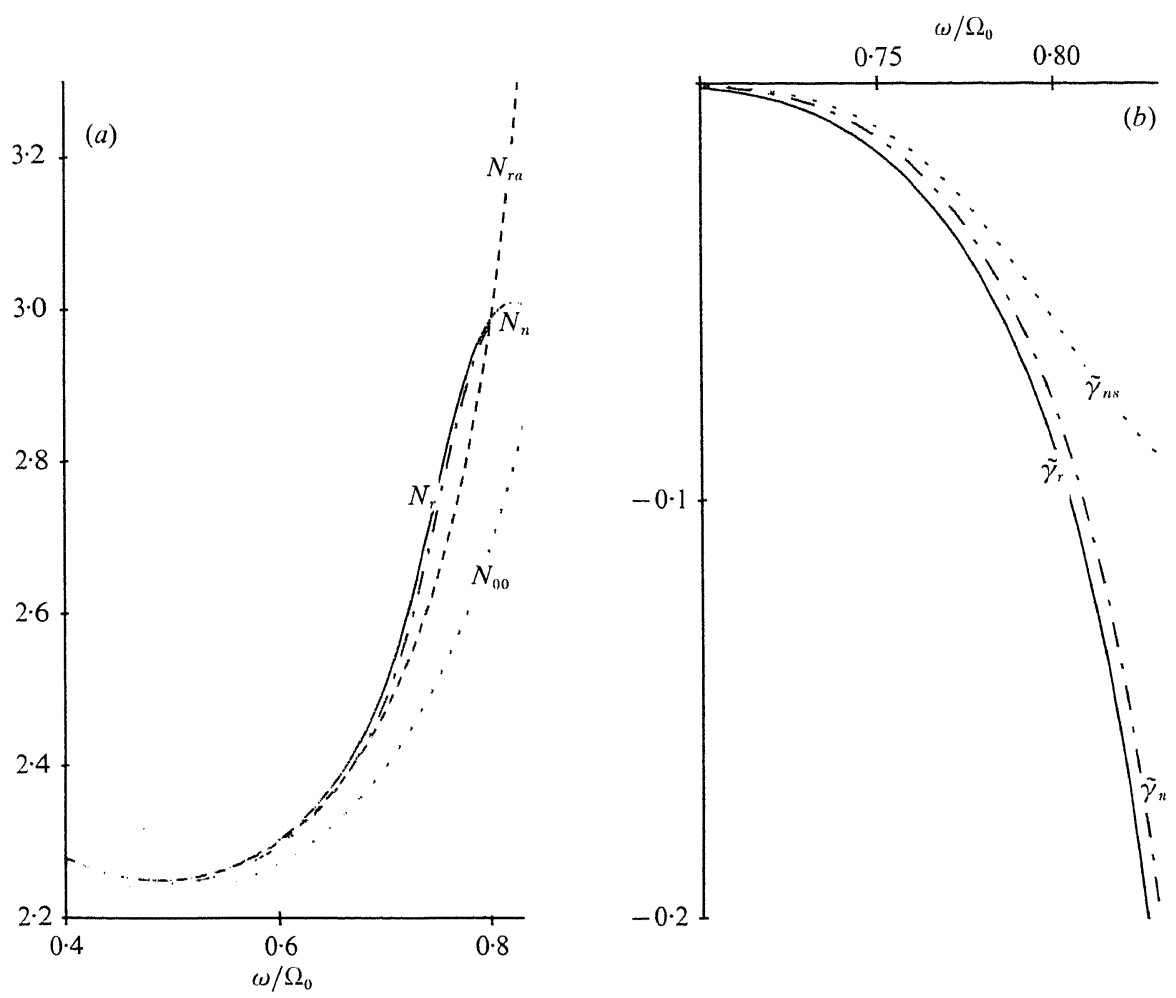

Frgure 5. As figure 4, but for $A_{e}=1$.

but the deviation between relativistic and non-relativistic curves is substantially larger compared with the case of $v=1$ and $v=5$. Relativistic theory predicts stronger instability, but at a lower frequency. The frequency of marginal stability is approximately $0 \cdot 457 \Omega_{0}$, which is again consistent with the results of the asymptotic analysis by Sazhin \& Temme (1991b).

In figure $4(a, b)$ we show curves similar to those in figure $2(a, b)$ but for a cooler plasma $(r=0.004$, which corresponds to electron energies of about $1 \mathrm{keV})$. As follows from figure $4(a)$, at $\omega / \Omega_{0} \lesssim 0.65$ the curve $N_{r a}$ practically coincides with $N_{r}$, but at larger frequencies the curve $N_{n}$ is closer to $N_{r}$ than the curve $N_{r a}$. The closeness between the curves $N_{00}$ and $N_{r}$ increases when $r$ decreases, which reflects the obvious result that a decrease in electron temperature makes the cold-plasma approximation more appropriate.

The curves $\tilde{\gamma}_{n s}$ and $\tilde{\gamma}_{n}$ shown in figure $4(b)$ coincide to within the accuracy of plotting. As in the case of the previously considered values of the parameters, the relativistic theory predicts stronger instability, but in a narrower frequency range. The absolute values of $\tilde{\gamma}$ are about 13 orders of magnitude smaller for $r=0.004$ than for $r=0.01$, which reflects the strong dependence of the increment of whistler-mode instability on electron temperature. At the same time, the frequency of marginally stable waves, $\omega \approx 0 \cdot 474 \Omega_{0}$, is close to that which follows from the computations for $r=0.01$. Again this is consistent with the asymptotic results of Sazhin \& Temme $(1991 b)$ (see their equation (20)).

In figure $5(a, b)$ we show curves similar to those in figure $4(a, b)$, but for an isotropic plasma $\left(A_{e}=1\right)$. The curve of $N_{r a}$ versus $\equiv \omega / \Omega_{0}$ is close to the curve 


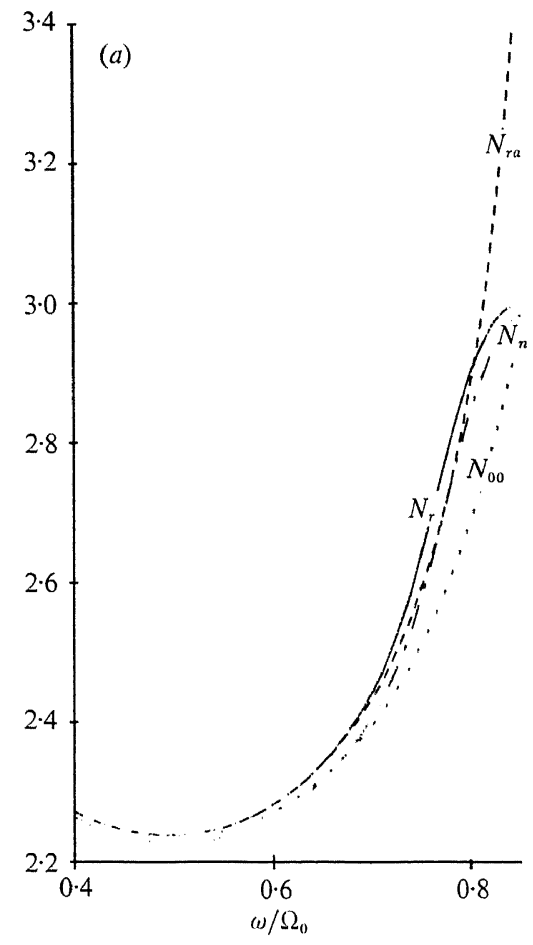

$\times 10^{-7}$

Figure 6. As figure 4, but for $A_{e}=3$.

of $N_{r}$ versus $\omega / \Omega_{0}$ only at $\omega / \Omega_{0} \lesssim 0 \cdot 6$. At larger $\omega / \Omega_{0}$ the non-relativistic approximation $N_{n}$ to $N_{r}$ gives better results. The derivation between the curves $N_{00}$ and $N_{r}$ is noticeably larger for $A_{e}=1$ than for $A_{e}=2$, which shows that the electron anisotropy tends to compensate for the effects of non-zero electron temperature on parallel whistler-mode propagation (cf. Sazhin, Ponyavin \& Varshavski 1979).

In the case of an isotropic plasma we have wave damping only, and this damping increases rapidly with increasing frequency. As follows from figure $5(b)$ the non-relativistic expression $\tilde{\gamma}_{n}$ gives a reasonably good approximation to $\tilde{\gamma}_{r}$, while $\tilde{\gamma}_{n s}$ substantially underestimates the values of $\tilde{\gamma}_{r}$ at frequencies close to the electron gyrofrequency. This result is consistent with the earlier result of Sazhin (1990).

In figure $6(a, b)$ we show curves similar to figure $4(a, b)$, but for a strongly anisotropic plasma $\left(A_{e}=3\right)$. The shapes of the curves in figure $6(a)$ are generally similar to those in $4(a)$ except that the approximation $N_{r a}$ to $N_{r}$ gives somewhat better results than $N_{n}$ at higher frequencies. At the same time, the increase in $A_{e}$ appears to have a large effect on the development of the instability. The increase of $A_{e}$ from 2 to 3 increases the range of instability by about $30 \%$, and increases the maximum values of $\tilde{\gamma}_{r}$ and $\tilde{\gamma}_{n}$ by about 17 orders of magnitude. As in the case shown in figure $4(b)$, the relativistic theory of whistler-mode instability for $A_{e}=3$ predicts a narrower frequency range of instability compared with the non-relativistic theory. However, the maximum values of $\tilde{\gamma}_{r}$ and $\tilde{\gamma}_{n}$ are roughly equal for $A_{e}=3$, the maximum value of $\tilde{\gamma}_{n s}$ being slightly larger than that of $\tilde{\gamma}_{n}$. 


\section{Conclusions}

Direct comparison between the results of numerical and analytical analysis for the parallel whistler-mode refractive indices $N$ and the increments of instability (or decrements of damping) $\gamma$ in weakly relativistic and nonrelativistic approximations brings us to the following main conclusions:

(i) The expression (15) for $N$ is the best approximation for numerical values of $N$ obtained in a weakly relativistic limit at relatively low frequencies (roughly $\omega / \Omega_{0} \equiv Y^{-1} \lesssim 0 \cdot 6$ ), but it breaks down rapidly as the wave frequency approaches the electron gyrofrequency (roughly $\omega / \Omega_{0} \gtrsim 0 \cdot 6$ ).

(ii) The non-relativistic expression for $N$ following from (12) can be used for qualitative analysis of whistler-mode propagation in a weakly relativistic plasma. This expression is the best approximation to the corresponding weakly relativistic expression when the wave frequency is closer to the electron gyrofrequency, provided that the condition (9) is satisfied.

(iii) Relativistic effects lead to a decrease in the frequency range of instability, in agreement with the earlier results of Sazhin \& Temme $(1991 a, b)$.

(iv) For moderate values of the anisotropy $\left(T_{\perp} / T_{\|} \approx 2\right)$ relativistic effects lead to an increase in the maximum value of the increment of instability $\left(\gamma_{\max }\right)$ : $\gamma_{\max }$ increases rapidly with increasing electron temperature and anisotropy.

(v) The expression (16) for the increment of whistler-mode instability (or the decrement of damping) is a good approximation to the non-relativistic expression (13) when $\gamma>0$, i.e. when we have instability; the expression (16) predicts weaker damping of whistler-mode waves at frequencies close to the electron gyrofrequency compared with the prediction of (13).

One of the authors (S.S.) would like to thank the staff of CWI and SARA for their hospitality and support during his stay in Amsterdam in October 1991, when the major part of this paper was completed. He is grateful to Dr Frans Vitalis and Mr Frank van der Wiel for their help in assessing computer facilities of CWI and SARA, and would like to thank NERC, the Netherlands Organization for Scientific Research (NWO) and the British Council for financial support of this project.

\section{REFERENCES}

Baynham, A. C. \& Boardman, A. D. 1971 Plasma Effects in Semiconductors: Helicon and Alfvén Waves. Taylor \& Francis.

Bullough, K. 1983 Space Sci. Rev. 35, 175.

Carpenter, D. L. 1988 Rev. Geophys. 26, 535.

Coroniti, F. V., Kurth, W. S., Scarf, F. L., Krimigis, S. M., Kennel, C. F. \& Gurnett, D. A. 1987 J. Geophys. Res. 92, 15, 234.

Gurnetr, D. A., Kurth, W. S., Cairns, I. H. \& Granroth, L. J. 1990 J. Geophys. Res. 95, 20,967 .

Helliwell, R. A. 1965 Whistlers and Related Ionospheric Phenomena. Stanford University Press.

JACQUinot, J. \& Leloup, C. 1971 Phys. Fluids 14, 2440.

Kaner, E. A. \& Skobov, V. G. 1971 Plasma Effects in Metals: Helicon and Alfvén Waves. Taylor \& Francis.

Kennel, C. F. \& Petschek, H. E. 1966 J. Geophys. Res. 71, 1. 
Robinson, P. A. 1986 J. Math. Phys. 27, 1206.

Robinson, P. A. 1987 a J. Plasma Phys. 37, 149.

Robinson, P. A. $1987 b$ J. Math. Phys. 28, 1203.

Sazhin, S. S. 1982 Natural Radio Emissions in the Earth's Magnetosphere. Nauka (in Russian).

Sazhin, S. S. 1987 a J. Plasma Phys. 37, 209.

Sazhin, S.S. 1987 b J. Plasma Phys. 38, 301.

SAZHIN, S. S. 1988 a Planet Space Sci. 36, 1111.

Sazhin, S. S. $1988 b$ J. Atmos. Terr. Phys. 50, 51.

SAZHIN, S. S. 1989 Physica Scripta 40, 114.

SAZHIN, S. S. 1990 Astrophys. Space Sci. 172, 235.

SaZHin, S. S. 1991 Ann. Geophys. 9, 690.

Sazhin, S. S., Ponyavin, D. I. \& Varshavski, S. P. 1979 Radio Phys. (Radiofizika) 22, 789 (in Russian).

Sazhin, S. S. \& Temme, N. M. 1990 Astrophys. Space Sci. 166, 301.

Sazhin, S. S. \& Temme, N. M. 1991 a Ann. Geophys. 9, 30.

Sazhin, S. S. \& Temme, N. M. 1991 b Ann. Geophys. 9, 304 (Erratum: p. 500).

Shkarofsky, I. P. 1966 Phys. Fluids 9, 561.

Storey, L. R. O. 1953 Phil. Trans. R. Soc. Lond. A 246, 133.

Temme, N. M., Sumner, A. E. \& Sazhin, S. S. 1992 Astrophys. Space Sci. (in press).

TsaI, S. T., WU, C. S., WANG, Y. D. \& Kang, S. W. 1981 Phys. Fluids 24, 2186.

Wharton, C. B. \& Trivelpiece, A. W. 1966 Plasma Physics in Theory and Application (ed. W. B. Kunkel), p. 233. McGraw-Hill.

Winglee, R. M. 1983 Plasma Phys. 2, 217. 